\title{
A semigroup with a right zero equal to a right identity is trivial
}

\author{
Open Mathematics Collaboration*†
}

June 27, 2020

\begin{abstract}
We prove the proposition addressed in the title of this paper.
\end{abstract}

keywords: semigroup, right zero, right identity, trivial

The most updated version of this paper is available at https://osf.io/zpwb5/download

\section{Notation \& Definition}

1. $[1,2]$

2. $\mathcal{S}=$ semigroup

3. $(\forall x \in \mathcal{S}: x z=z) \rightarrow(z=$ right zero $)$

4. $(\forall x \in \mathcal{S}: x e=x) \rightarrow(e=$ right identity $)$

5. $(\exists ! x \in \mathcal{S}) \leftrightarrow(\mathcal{S}=$ trivial $)$

*All authors with their affiliations appear at the end of this paper.

†Corresponding author: mplobo@uft.edu.br | Join the Open Mathematics Collaboration 


\section{Proposition}

6. If $\mathcal{S}$ is a semigroup and $e \in \mathcal{S}$ is both a right zero and a right identity, then $\mathcal{S}$ is trivial [1].

\section{Proof}

7. Since $e$ is a right zero, then $\forall x \in \mathcal{S}: x e=e$

8. Since $e$ is a right identity, then $\forall x \in \mathcal{S}: x e=x$

9. From (7) and (8), $x e=x e$, then $x=e$ for all $x \in \mathcal{S}$.

10. Therefore, $\exists ! x \in \mathcal{S}$.

\section{Final Remarks}

11. $((\mathcal{S}=$ semigroup $) \wedge(e \in \mathcal{S}) \wedge(\forall x \in \mathcal{S}: x e=e=x)) \rightarrow(\exists ! x \in \mathcal{S})$

\section{Open Invitation}

Review, add content, and co-author this paper $[3,4]$. Join the Open Mathematics Collaboration (https://bit.ly/ojmp-slack). Send your contribution to mplobo@uft.edu.br.

\section{Open Science}

The latex file for this paper together with other supplementary files are available [5]. 


\section{Ethical conduct of research}

This original work was pre-registered under the OSF Preprints [6], please cite it accordingly [7]. This will ensure that researches are conducted with integrity and intellectual honesty at all times and by all means.

\section{References}

[1] Cain, Alan J. Nine Chapters on the Semigroup Art. AJC Porto \& Lisbon, 2020. http://www-groups.mes.standrews.ac.uk/\%7ealanc/teaching/m431/

[2] Lobo, Matheus P. "Open Mathematics Knowledge Base." OSF Preprints, 13 May 2020. https://doi.org/10.31219/osf.io/evq8a

[3] Lobo, Matheus P. "Microarticles." OSF Preprints, 28 Oct. 2019. https://doi.org/10.31219/osf.io/ejrct

[4] Lobo, Matheus P. "Simple Guidelines for Authors: Open Journal of Mathematics and Physics." OSF Preprints, 15 Nov. 2019. https://doi.org/10.31219/osf.io/fk836

[5] Lobo, Matheus P. "Open Journal of Mathematics and Physics (OJMP)." OSF, 21 Apr. 2020. https://doi.org/10.17605/osf.io/6hzyp

[6] COS. Open Science Framework. https://osf.io

[7] Lobo, Matheus P. "A Semigroup with a Right Zero Equal to a Right Identity Is Trivial." OSF Preprints, 27 June 2020. https://doi.org/10.31219/osf.io/zpwb5 


\section{The Open Mathematics Collaboration}

Matheus Pereira Lobo (lead author, mplobo@uft.edu.br) $)^{1,2}$

${ }^{1}$ Federal University of Tocantins (Brazil); ${ }^{2}$ Universidade Aberta (UAb, Portugal) 